\title{
Idea on Holistic Medicine Based on the Electron Leak Pathways of Mitochondrial Respiratory Chain
}

\author{
Xu Jianxing \\ Institute of Biophysics, Chinese Academy of Science, Beijing, China
}

Email address:

xujx@sun5.ibp.ac.cn

To cite this article:

Xu Jianxing. Idea on Holistic Medicine Based on the Electron Leak Pathways of Mitochondrial Respiratory Chain. American Journal of Clinical and Experimental Medicine. Vol. 7, No. 6, 2019, pp. 135-142. doi: 10.11648/j.ajcem.20190706.13

Received: September 18, 2019; Accepted: October 21, 2019; Published: December 13, 2019

\begin{abstract}
With the development of physical technology, life science has reached the molecular level. With the development of molecular biology, western medicine has carried out in-depth analysis and Experimental Research on diseases. Nevertheless, wise thinking about the theory and current situation of Western medicine can still find some problems worth to investigation. With the breakthroughs in cell and molecular biology, protein function and gene regulation have become the starting point and theoretical basis for thinking about medical problems and developing drugs. However, the human body is a multicellular organism, which is the coordination of multiple functional organs formed by orderly accumulation of cells. At present, the knowledge of molecular biology on the orderly accumulation of cells is still scarce in life science. This knowledge black hole makes Western medicine far from being based on a comprehensive understanding on the human body, thus making the scientific nature of Western medicine imperfect. Mitochondrial symbiosis is a landmark event in which life evolves from a single cell to a multicellular one. In our lab the discovery of electron leak pathways of the mitochondrial respiratory chain reminds me to describe the function of respiratory chain as the molecular "life-engine". The role of ROS generated in mitochondria through the electron leak pathways of the respiratory chain has become a new investigative field of health and longevity. The signal effect of ROS on antioxidant activity and tissue repair activity has become a hotspot in medical research. An idea to explore medical problems in perspective of bioenergetics came into being in my mind. In 2016, I published a paper in the Journal of clinical and experimental cardiology, which suggested that the human body should be regarded as a whole composed of a huge number of "life engines", and human health and medicine should be studied with thermodynamics. A big question is that the ROS generated by the electron leakage of respiratory chain has two opposite roles: it causes aging and diseases by the oxygen toxicity of ROS and it role as signal to stimulate antioxidative activity or tissue repair ability. Then how the human body makes decision to tell ROS play as a positive or negative role? Answering this question takes me long time searching. In this paper, I will elaborate on this issue from the perspective of cosmic energetics.
\end{abstract}

Keywords: Respiratory Chain, Life-engine, Vitality, Health and Longevity

\section{Introduction}

The structure and function of respiratory chain have been studied for nearly a hundred years. As we all know, Madame Curie discovered radium and radioactivity, opened up new fields of radiochemistry and atomic energy research, and won two Nobel Prizes. But few people know that D. Keilin discovered three cytochromes related to cell respiration. He was contemporary with Curie and used the same spectral analysis method. He also opened up a new field of bioenergy. However, because biology was far more complex than chemical physics, he did not receive the Nobel Prize, but left the chance to succeed bioenergetics. In 1961, P. Mitchell put forward the theory of chemical osmosis to describe the mechanism of ATP produced by electron transfer in the respiratory chain. After more than ten years of experimental testing, he won the Nobel Prize in 1978. Also in 1961, Boyer put forward the conformation theory describing the mechanism of ATPase synthesis ATP and won the Nobel Prize in 1996.

My three tutors, Wang Y. L, Tsou C. L. and Tsoo E. King, are all students of Keilin and have made outstanding contributions to the early study of respiratory chain structure. Wang Y. L was the first person to determine that flavin is also 
involved in the electron transfer function of cytochrome chains. Tsou C. L was the first person to estimate the role of cytochrome $\mathrm{c}$ by the method of extraction and reconstitution of cytochrome $\mathrm{c}$ from the respiratory chain, and late on working with Wang Y. L did the extraction and reconstitution of succinate dehydrogenase. Tsoo E. King continued to work in the United States. He decomposed and reorganized the whole respiratory chain. Their work identifies the electron transport pathways of the respiratory chain and provides the basic knowledge for Mitchell's theory. My laboratory was established by these three elders in 1978. We published the experimental evidence of electron leakage bypass in the respiratory chain in 2003. Four electron leakage pathways have been declared free radical metabolism in mitochondria.

The discovery of electron leakage bypass of the respiratory in our laboratory not only provided an explanation on the deviation of $\mathrm{P}$. Mitchell's theoretical calculation and experimental data but also makes us realize that the function of the respiratory chain is actually a "life-engine" on the molecular scale. Engine burns oil to generate power driving mechanical operations. As a "life-engine", the respiratory chain is burning grain to produce ATP, which is the energy carrier molecule driving the vitality of life. This inspired me to establish a new medical principle with respiratory chain function as the core.

To explore human health problems, we need not only to understand the molecular biological processes of cells, but also to understand how cells and cells accumulate in an orderly manner to successfully coordinate the whole. What forces and mechanisms promote orderly accumulation of cells is more important knowledge for human health studies, but little is known about it. Mitochondrial symbiosis is the landmark event in the evolution of organisms from single-cell to multi-cellular organisms. The information of orderly accumulation of cells must be included in the interaction between mitochondrial gene and chromosome genes. At present, there are more studies on the molecular biology of protein function and cell gene expression, but less on the molecular biology of glycoproteins and polysaccharides. The role of polysaccharides and glycoproteins in the orderly accumulation of plant cells can be clearly seen. Animals and plants follow the same rule in the orderly accumulation mechanism of cells. The complexity of polysaccharide branched chains interconnection enables polysaccharide molecules to form different morphological structures such as membrane, pipe and lattice. Lattice structure is the cell wall of plants. The spatial arrangement of lattices places cells in specific spatial positions. Pipe structure can form logistics network. Polysaccharide membrane structure can wrap cells and tissues to form organs and connect different organs to form complex human body structure. A large number of conjugated double bonds in polysaccharide molecules may play an important role in information transmission between cells, which is equivalent to the information network of human cells. Before the molecular biological mechanism of orderly accumulation of cells in human body is fully understood, it may be a shortcut to explore this issue from the perspective of bioenergetics. Respiratory chain is a branch of life science from the perspective of energy. The discovery of electron leakage bypass in the respiratory chain prompted me to conceive a holistic medical principle based on the function of mitochondrial respiratory chain.

\section{Four Electron Leak Pathways of Mitochondria Respiratory Chain}

The discovery of electron leak pathways of mitochondrial respiratory chain reveals that the respiratory chain not only involved in ATP synthesis through the electron transfer pathway but also involved in ROS generation through the electron leak pathways [1-3]. Four pathways of $\mathrm{O}_{2}^{-\cdot}$ linked to the electron leakage of respiratory chain can be defined as the radical metabolism of mitochondria as shown in figure 1 .

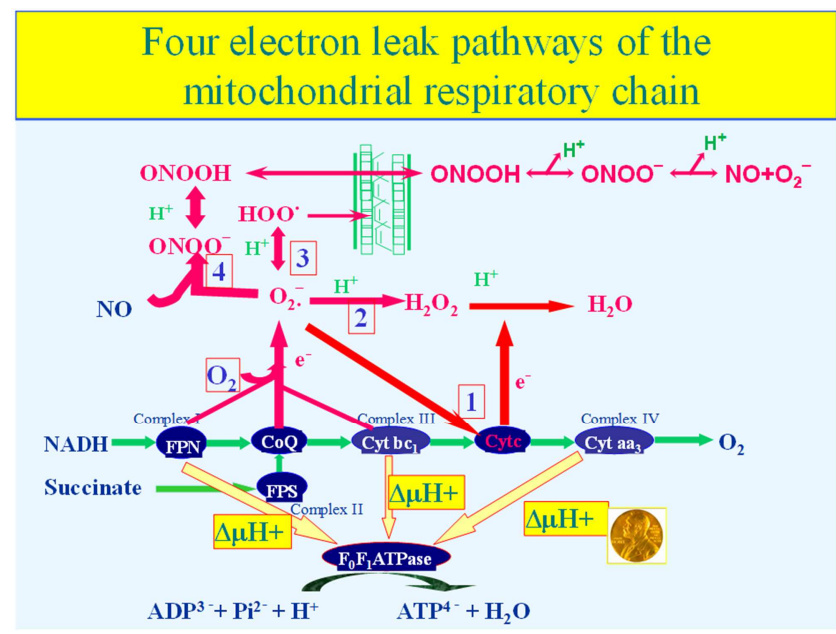

Figure 1. The dark cycles are the enzymes involved in ATP synthesis of mitochondria. Green color arrows show the way of electron transfer in the respiratory chain. Yellow color shows the Mitchell's theory on the coupling mechanism of respiratory chain and ATPase. The red colored part shows the four electron leak pathways established in our lab. As the involvement of reductive route $\left(\mathrm{O}_{2} \rightarrow \mathrm{O}_{2-} \cdot \rightarrow \mathrm{H}_{2} \mathrm{O}_{2} \rightarrow \mathrm{H}_{2} \mathrm{O}\right)$, the electron leakage is defined as the radical metabolism partner to ATP production. Oxygen consumed in the electron leakage is not used in ATP production but spent in the generation of reactive oxygen species ( $\mathrm{ROS}$ ) such as $\mathrm{O}_{2-} . \mathrm{H}_{2} \mathrm{O}_{2} \mathrm{HOO} \cdot$ and $\mathrm{ONOO}$.

Two (1) (2) of the cytochrome $c$ mediated electron leak pathways of respiratory chain has been realized as a self-defense of respiratory chain against $\mathrm{O}_{2}^{-\cdot}$ and $\mathrm{H}_{2} \mathrm{O}_{2}$ [1-5]. The way of $\left(\mathrm{O}_{2}^{-}+\mathrm{H}^{+} \rightarrow \mathrm{HOO}\right)$ (3) could be involved in body temperature according to the investigation of Bielski's on the exothermic reaction of $\mathrm{HOO}$ with double alleles $\mathrm{H}$ atom of unsaturated lipid in membrane [6]. The way of $\left(\mathrm{O}_{2}^{-}+\right.$ $\mathrm{NO} \rightarrow \mathrm{ONOO}^{-}$) (4) could have role in the regulation of ATP synthesis because NO bonding to the reactive center of complex IV hinder the production of ATP, whereas $\mathrm{O}_{2}^{-}$ bonding with $\mathrm{NO}$ recover the NO hindered ATP production. The way of $\left(\mathrm{O}_{2}^{-}+\mathrm{NO} \rightarrow \mathrm{ONOO}^{-}\right)$(4) can also help $\mathrm{O}_{2}^{-}$and NO penetrate membranes for long distance diffusion, the physiological role of this diffusion is worth to investigate. The involvement of ROS in the four electron leak pathways inspire a new thought on the function of the respiratory chain. 


\section{The Biomedical Significance of the Radical Metabolism of Mitochondria}

The four electron leak pathways can be defined as the radical metabolism of mitochondria. Then the mitochondria has two fractions of oxygen consumption: (1) the oxygen consumed in the ATP synthesis is called phosphorylative respiration, which is completed through the electron transfer of the respiratory chain and obeys Mitchell's theory; (2) the oxygen consumed in ROS generation can be called nonphosphorylative respiration, it is a side reaction associated with ATP production. The nonphosphorylative respiration is less than $2 \%$ of the total oxygen consumption in the normal physiological condition according to the data of Chance's group [7]. Whereas in our lab it is observed that the nonphosphorylative respiration can be higher when animal in pathological conditions $[4,5]$. Therefore the nonphosphorylative respiration might have more complicated biological significance. The ROS generated in the electron leak pathways should have physiological function when in lower normal concentration. The higher than the normal concentration of ROS would play as a warning signal to stimulate cell producing more antioxidant enzymes to remove the over generated toxic ROS. Continuous increase of ROS would cause cell aging, apoptosis and death. In this consideration, the higher electron leakage of the respiratory chain should have a pathological significance. We studied the role of ROS in Parkinson's disease and ischemia/reperfusion injury by controlling the electronic leakage of respiratory chain, and clarified the role of electronic leakage of respiratory chain in the pathogenesis [8-11]. The ratio of the four metabolic pathways of the respiratory chain in mitochondria could also have implications in the metabolic types of different persons.

\section{Biological Evolution of the Respiratory Chain}

In answering why the respiratory chain has the electron leak pathways, we published a paper to announce that the electron leak pathways were the evolutional event of organisms against oxygen toxicity shown as in figure 2 [12].

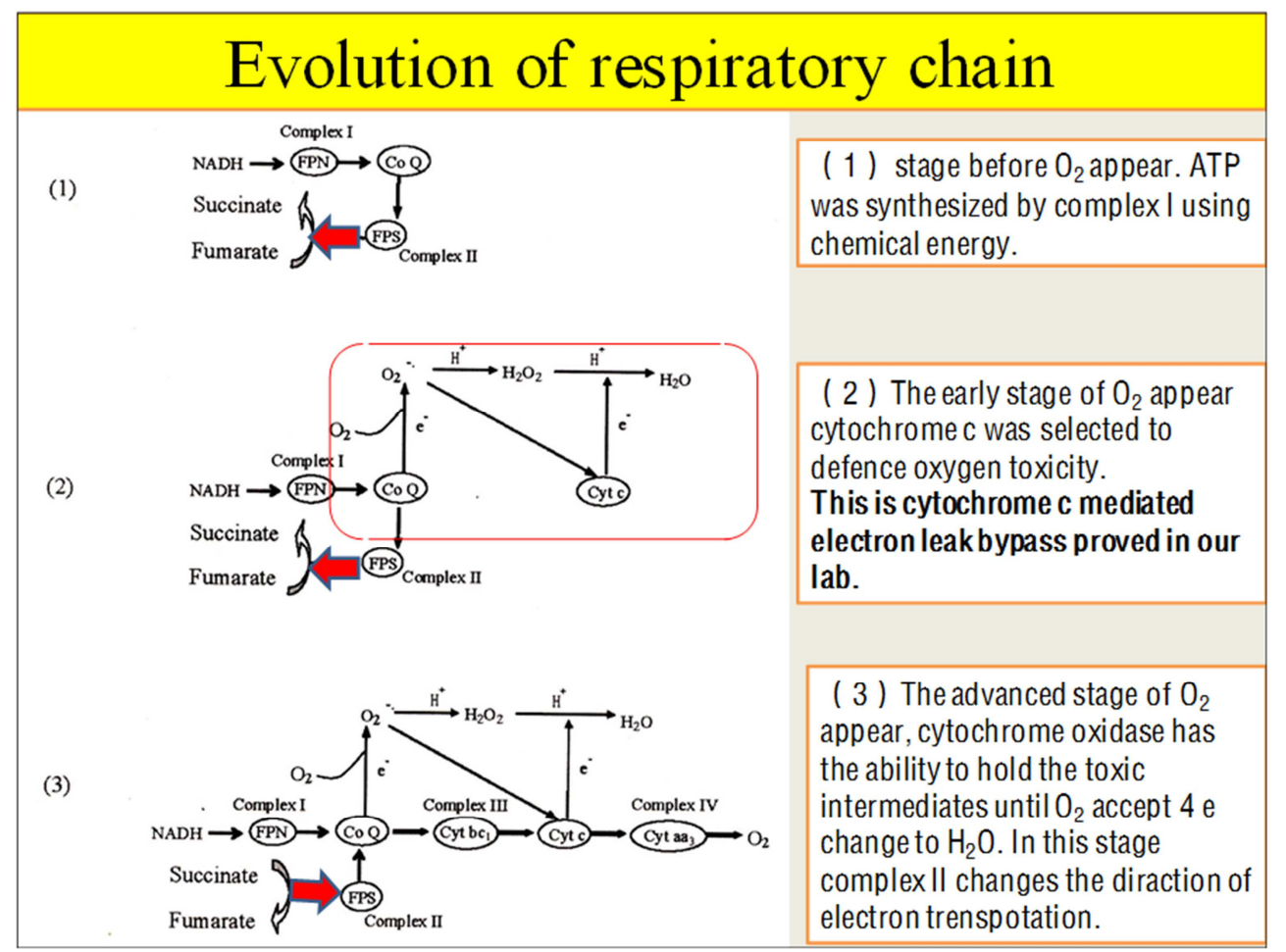

Figure 2. The red square shown in (2) is the two electron leak pathways mediated by cytochrome c proved in our lab. It suggests the appearance of the detoxify event in the early stage of oxygen. The highly evolved respiratory chain, shown in (3), has a big change in the direction of the electron transfer in complex II. The experimental evidence is performed in the purified complex II [12]. The normal electron transfer of complex II is from succinate to cytochrome $b_{560}$, once the oxygen in the reactive medium is replaced by argon, the electron transfer reversed from cytochrome $b_{560}$ to succinate.

It is well known that in the early stage of the earth there was no oxygen in the atmosphere. Reduced atmosphere was beneficial for the accumulation of the organic molecules, and the concentrated organic chemicals in the marine wetlands incubated the forms of original life. The later appearance of oxygen let the original life face serious damage of oxygen toxicity. Survivors have to develop the ability to fight with oxygen toxicity. The respiratory chain is the evolved event of the living organisms to defend against oxygen toxicity. The cytochrome $\mathrm{c}$ mediated two electron leak pathways proved experimentally in our lab reflects the early stage of detoxification. Facing the strong power of oxygen toxicity, the evolved life only can select the way of generation to generation for survival. Then we can think of aging as the 
history of one generation of living body fighting with oxygen toxicity and the longevity as the stronger detoxifying ability or less toxic damages. The warning is that today's planet is not only aerobically toxic, but more industrial pollution complicates the research on health and longevity. The ROS generated in the electron leak pathways of the respiratory chain can be a dangerous factor in the human body for causing oxidative damager and leading to disease or aging, but more important is that the ROS in mitochondria play as the signals to stimulate anti-oxidative function and strengthen the ability of repairing on the damage. The latter is the theoretical base of health and longevity. Then the physiological role of different electron leak pathways of the respiratory chain would be more important for the investigation of health and longevity.

\section{The Role of Coenzyme $Q_{10}$ in Heart Nursing and Health Care}

$\mathrm{Q}_{10}$ is the linkage of complex I II and III. Both $\mathrm{Q}_{10}$ and cytochrome c performed key role in saving life from the disaster of oxygen toxicity in the early stage of life evolution in earth. In this consideration, the role of $\mathrm{Q}_{10}$ in health care is definite [12]. $\mathrm{Q}_{10}$ has been purified and used in heart care clinically in the 1960 's. The problem is that the clinical efficacy can only be seen at high doses, but oral administration of high doses of $\mathrm{Q}_{10}$ can have side effects on the stomach. The finding of electron leakage of the respiratory chain revealed that the ROS generated in the electron leak pathways is the reason for causing the side effect. The electrons leaked into the inside of the inner membrane of mitochondria let the mitochondria DNA in higher rate of ROS risk, whereas the electrons leaked to the outside of the inter membrane let the cytochrome $\mathrm{c}$ in dilemma of scavenge ROS and transfer electrons [12]. Therefore an idea on cocktail of $\mathrm{Q}_{10}$ is suggested for the clinical use as shown in the figure 3 .

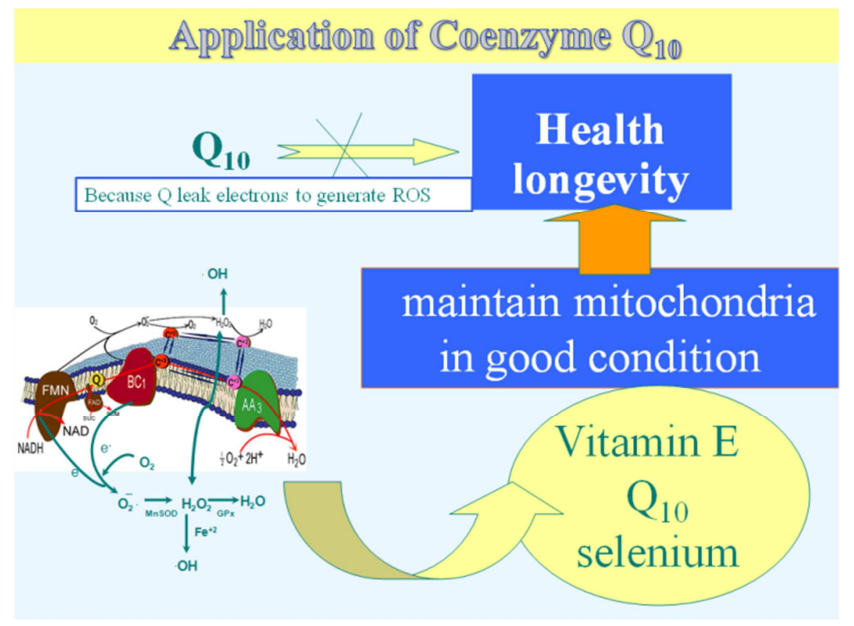

Figure 3. the cocktail of $Q_{10}$ suggested in clinical use.

We announced a patent in 2000 suggested that the scavenger, such as Vitamin E, can decrease the ROS damage on the local absorptive cells before $\mathrm{Q}_{10}$ spreading (ZL 2000 00102763.8). In 2009 a patent suggested that an cocktail of $\mathrm{Q}_{10}$ Vitamin E and Selenium can be used clinical to prevent heart from disease for Selenium protecting mDNA from ROS damage by increasing activity of GSH-peroxides (ZL 20091 0085018.5).

\section{Respiratory Chain Functions Like a Molecular "Life-engine"}

In view of thermodynamics, the respiratory chain can be conceptualized as a molecular "life-engine". The function of respiratory chain to produce ATP by oxidizing nutriment is similar as an engine producing energy by burning petroleum. According to the principle of thermodynamics, an engine spends energy to drive machine doing "useful work" is always associated by spending energy to overcome mechanical friction doing "idle work". Doing "idle work" is the reason for causing aging of the machine, but the respiratory chain as a "life-engine" has more biological significance in its "idle work". The ROS generated from the electron leakage of the respiratory chain has two functions: (1) the damaging effect of ROS that causes cell to age, this is similar to the non-life engine; (2) the ROS as a biological signal to stimulate defense and repairs ability, this is significantly difference from the "idle work" of a non-life engine. The defense ability is to remove the toxic ROS before the damage occurs, and the repairing ability is to regenerate the ROS damaged molecules, such as proteins, nucleic acid and lipids, etc. Both abilities are important for human body's health and longevity. The ROS generation in mitochondria through the electron leak pathways of respiratory chain inspires us to investigate health and longevity with the theory of thermodynamics.

\section{Bioenergetics' Principle of Health and Longevity}

The discovery of electron leak pathways of the mitochondrial respiratory chain inspires us to investigate human health with a new insight of bioenergetics. The human body is exactly depending on the numerous of "life-engine" to supply energy. How to maintain "life-engine" in good working condition is the key point on the research of health and longevity. Taking the consideration of the human body as a thermodynamic machine driven by a huge numbers of life-engine, the thermodynamic theory can be applied for the theoretical base of holistic medicine.

Human body is a very complicated and highly organized system. Entropy is the term of thermodynamics to describe the orderliness, the increase of entropy means more chaotic, the decrease of entropy means more orderly. Nobel Prize winner Ilya Prigogine introduced a concept of dissipative structure in 1977 , it is a thermodynamic theory to describe the organizing process of material from its chaos disorder. The dissipative structure must be satisfied by two requirements: one is continual producing "negative entropy" 
by exchanging energy with the environment, and another is keeping the organized structure continual renew by exchanging material with the environment. Once any one of the two conditions is not satisfied the order of the dissipative structure would be lost and the highly organized dissipative structure would be destroyed.

If using the thermodynamic dissipative structure to describe the organization of human body, then the orderliness can be used as a measure on the health of the human body. Pregnancy can be thought of as the constructive process of dissipative structure under the control of gene regulation. The inborn orderliness can be thought as the health capital inherited from the parents. Natural orderliness varies from person to person and it is not perfect. The shortage of the inborn orderliness is the weak point of gene; it is come from the defection of parent's genes. The defect is always well covered during embryonic development, not well covered defection is the children born with congenital genetic diseases.

The huge numbers of life-engine is the key of the human body exchange energy and material with the environment. They play an important role in the maintenance of the dissipative structure of the human body. Once life-engine becomes damaged or aged, the highly ordered human body would go down to disorder, that is aging and diseases. Then we can define health and longevity in terms of thermodynamics: the health is the maintenance of the inherent orderliness; the longevity is the history of human body fighting to damaging factors for the maintenance of the inherent orderliness. The sub-health can be thought of as different level of damaged statement of the orderliness. The recovery of health of the human body can be thought of as the repairing of the damaged orderliness. Thermodynamics is a state function, which is reversible. Sub-health is a state of confrontation between human and environmental harmful factors. The accumulation of sub-health leads to disease and aging. It is very important to repair sub-health in time.

\subsection{Dual Nature of the ROS Generated from the Electron Leak Pathways of Mitochondrial Respiratory Chain}

The fact is that the ROS generated in the electron leak pathways of mitochondrial respiratory chain have two opposite effects on the human body. The negative effect of ROS is causing the oxidative damage on the order of human body leading to disease and aging. The positive effect of ROS is playing as the signal to stimulate the antioxidative function and strengthen the ability of damage repairing, that is a reconstructive process of the order of dissipative structure of the human body.

The question is how the human body makes the decision telling ROS to play as the positive role or the negative role? How the judgment is made in human body?

There are two complex difficulties in answering this question.

One complexity is the communication between the chromosome DNA and the mitochondria DNA. The mutual communication of both DNA systems is the matter regarding to the evolution of life from single-cell life to multi-cellular organisms. There are only single-celled organisms appear on the earth before the symbiosis of mitochondria. This means that the health problem of human body is depending on both DNA systems but not single of chromosome DNA. The mutual communication between the two DNA systems is the base for the orderly organization of cells in the human body.

Another complexity is the communication between the two brains of human body: the inborn brain and the educated brain. The inborn brain is similar as animal brain, whereas the educated brain is equipped by knowledge during their growing up. The desires of the inborn brain are generated from the physiological needs, whereas the desires of the educated brain are generated from the social competition. The educated brain not only is the identification of the human body from animal, but also is different from person to person depending on their level of education and their different civilization of living. This complexity cannot be solved in animal model experiments of the western medicine research. The bioenergetics' principles of health and longevity presented above in the perspective of thermodynamics could be an ideology to overcome this complexity.

In order to explain the switching mechanism of ROS, the principles of cosmic energetic is figured out in my mind (see Figure 4). The concept of "energy entanglement" is introduced to describe the force of human body to construct and maintain the "orderliness" of cells. Energy entanglement emphasizes the existence of a force in the human body, which tightens all the cells tissue and organs that make up the human body into a complex and orderly whole. This force is the so-called vitality, which is the driving force of the origin and evolution of life, but also the driving force of individual development, exists in everyone's life.

\section{Principles of cosmic energetics}

The concept of energy entanglement is used to describe the object in nature. It is intended to emphasize the existence of energy entanglement force inside the object. It tightens all the elements that make up the object into a whole.

\begin{tabular}{|lll|}
\hline \multicolumn{2}{|c|}{ The following are different levels of energy entanglement and } \\
& entanglement forces \\
\hline Energy entanglement & $\underline{1}$ Entanglement force \\
Elementary particle & 1 & Atomic force \\
Chemical compound & 1 Chemical bond \\
Polymer Compounds & $\uparrow$ covalent bond \\
Biomacromolecule & $\uparrow$ Hydrophilic Hydrophobic Bonds \\
cell & $\uparrow$ (biomembrane) \\
multicellular organisms & $\uparrow$ (mitochondria) brain \\
Human body & 1 Intelligent Brain \\
society & 1 Social and Cultural Consciousness \\
\hline
\end{tabular}

Figure 4. Principles of cosmic energetics.

The evolution of matter from simple to complex, from inanimate to life, from single cell to multi-cell, from individual life to community society follows the principle of thermodynamic negative entropy. This is an evolutionary process that adds order and complexity. All complex ordered structures of different levels can be called energy 
entanglements. The force of energy entanglement can be atomic force, chemical bond, hydrophilic bond, hydrophobic bond, etc.

The idea of cosmic energetics comes from the Einstein's Formula $\mathrm{E}=\mathrm{mc}^{2}$. It is not a mathematic formula but Einstein's view on the universe. Everything in the universe is an entanglement of energy, just different in size and complexity. Energy is the force for the development of the complexity and orderliness of material, and material structure is also the carrier of energy. Every object has two attributes: (1) individual particularity $(\mathrm{m})$, which is material, has specific structure and properties, and (2) group field (E) is the correlation of energy and information between different individual particularities. Exploring anything can be done from the point of view of physical structure (m), or from the point of view of energy and information (E). From the perspective of energy and information, all objective objects can be called energy entanglement, but their energy entanglement fields can be gravitational field in astrophysics, electromagnetic field in microphysics, love field and reputation field in human society. According to the theory of cosmic energetics, the origin and evolution of life is a process of material structure increasing complex and orderly, and "vitality" is the power to construct orderly cells and maintain human health. This theory not only answers the mechanism of ROS switch, but also provides a theoretical basis for holistic medicine to explore health problems from the perspective of energy.

"The human body is a shrinking universe" is emphasizing that all levels of entanglement are exist in the human body. Entanglement force closely combines all levels of biological molecules, cells, tissues and organs into a highly complex and orderly system with highly automated management and the ability to self-regulate and repair damages. The functional combination of energy entanglement at all levels of human body system and its orderly harmony in space and time are the basis of health. The human body itself is the entanglement of a large number of cells, tissues and organs, and human is also a member of the entanglement of social groups. There should be ROS switch mechanism in the role conversion between two entanglements. The harshness of social competition brings complex emotions to people. Bad emotions can harm the harmony and order of individuals. Therefore if you are optimistic and positive, ROS will play an active role in overcoming sub-health, and your body will get better and better. If you are negative and pessimistic, ROS plays a negative role, your body will get worse and worse. A thousand miles are lost in one thought.

\subsection{The Natural Law of Health and Longevity}

Vitality is the driving force for the body to maintain the "inherent orderliness" from injury or rebuild the "damaged orderliness" to restore health. Vitality comes from the respiratory chain. Respiratory chains convert solar energy stored in food into energy carrier molecule ATP for human body use. ATP is the capital of life vitality. As long as the respiratory chain works properly, the vitality will repair the damage and restore health in time. The existence of vitality makes most sub-health status reversible, which is the theoretical basis of self-healing medicine.

How does the human body repair sub-health? This is a critical issue.

The concentration of oxygen molecules is the regulator of respiratory chain synthesis ATP. The concentration of oxygen molecules in blood changes periodically with one breath and one breath. The frequency of breathing and the rate of heartbeat are different. The superposition of two frequency waves will produce a periodic rhythm of blood oxygen concentration. The different time point of convergence of slow breathing wave and fast heart rate wave will generate different superposition of oxygen concentration wave. Therefore, each person establishes a unique time rhythm of blood oxygen concentration from the moment when cut the umbilical cord to open the alveoli starting breath. This periodic cycle of the time rhythm of blood oxygen concentration guides the orderly operation of the respiratory chains in different parts of the body. A healthy human body is like a symphony of multiple functional groups of the respiratory chain. The rhythm of oxygen concentration in the blood is the conductor of the symphony.

It must be remembered that the time rhythm of oxygen concentration is your own health code and your own best doctor. Every breath is a health code scanning on the working state of all the respiratory chains in different part of the body. It is also a functional debugging of the respiratory chains that do not operate in accordance with the normal rhythm. The human body itself is an energy entanglement with orderly accumulation of cells. Linked information feedback mobilizes all the forces of the human body to correct the local dysfunction. Therefore, adjusting the disorder of the body's local spatial structure with the time rhythm of the human body's own breathing is the natural law of restoring health. Hold the natural rhythm of breathing, let the body and mind "quiet" down, achieve "no ego" state, feel that breathing is sending energy to the lesion, repairing damage. This is the state in which vitality plays its role and also the scientific principle of Qigong.

In the struggle against harmful environmental factors, the "orderliness" of different levels of human body structure will lead to the overall fatigue and discomfort of human body once disturbed. Sleep is the best way to restore "orderliness" and eliminate fatigue and discomfort. In deep sleep, energy is consumed to repair damage in the body, which is the best time for vitality to play its role. When you wake up, your body stays alert and your energy is spent outside. Damages that fail to be repaired in time during sleep can accumulate to cause obsolete injuries and sub-health. People in pathological state feel uncomfortable when they sleep in shallow sleep, and waste energy with fantasy. At this time, you need to adjust your mind, build confidence in your own vitality, concentrate on your natural breathing rhythm, use energy to repair damages, sub-health pressure will gradually ease, and eventually return to a healthy state. 


\section{Jingluo (Meridian) Is the Rule of the Orderly Accumulation of Cells}

The above mentioned is a new idea of holistic medicine based on the discovery of electronic leak bypass of respiratory chain. The basic point is to understand the respiratory chain as a "life-engine" and the human body as a collection of huge number of "life-engines". If we regard "useful work" of "life-engine" as "yang" and "useless work" as "yin", then the respiratory chain can be understood as
"Yin-Yang element", and the human body can also be understood as a collection of a large number of "Yin-Yang elements". In this way, the view on holistic medical based on the function of respiratory chain fully conforms to the medical view of Yin-Yang regulation of traditional Chinese medicine. All the idea above mentioned based on the respiratory chain as the "life-engine" will be in agreement with the respiratory chain as the "Yin-Yang element" of Chinese medicine.

\section{"Life-engine" can be the Yin-Yang elements}

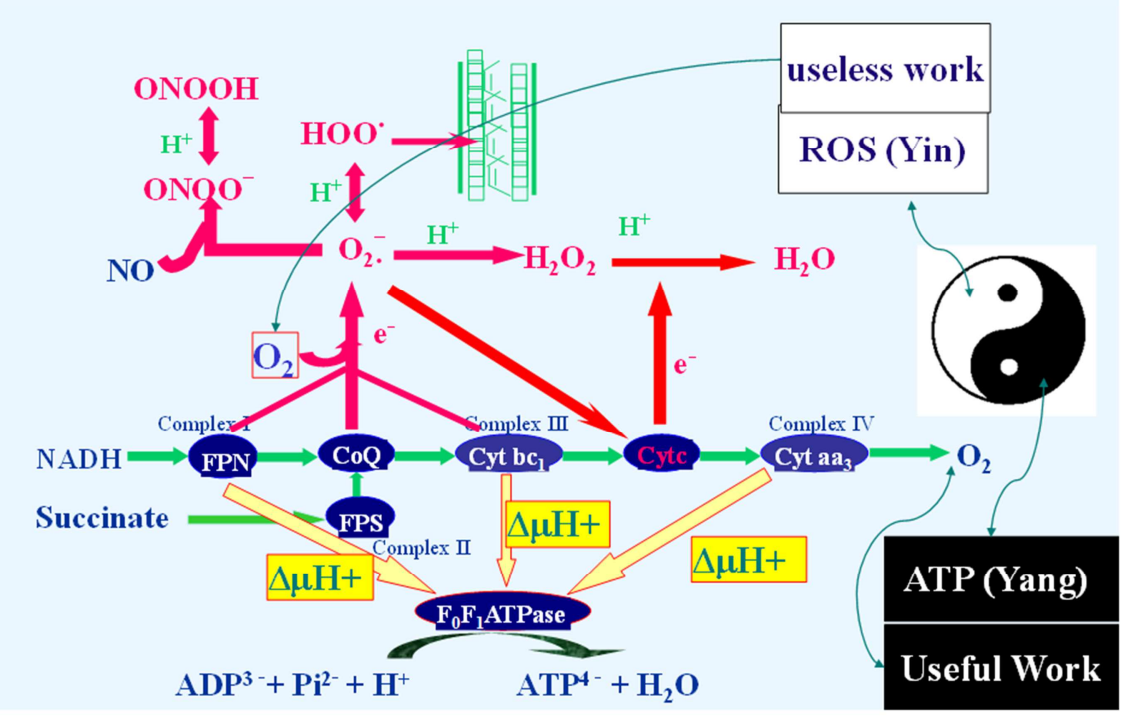

Figure 5. Respiratory chain as Yin-Yang elements of TCM theory.

The concept of "energy entanglement" is introduced, which emphasizes the close connection of all cells, tissues and organs to form a complex and orderly human body. This idea fully conforms to the concept of "Qi" in traditional Chinese medicine. Qi, like vitality, is the force that makes up the orderly accumulation of cells and keeps them in order. Chinese medicine regards the human body as an intrinsically complex and reasonable "black box", observes the output response of the external input signal, and analyses the physiological and pathological state of the human body from the correlation between input and output. Signal input refers to treatment options, such as prescription selection or acupuncture points selection. Signal output is the body surface manifestation caused by disease, such as tongue, pulse and so on. In the past 5000 years, a large number of patients directly observed the accumulation of curative effects; Chinese medicine has developed a systematic theory and treatment methods recorded in ancient Chinese medical works.

By observing the correlation between output and input, Chinese medicine took the lead in discovering the meridian system and the treatment of dredging meridians. The essence of meridians is the orderly accumulation of human cells, the kinship network of cell lineages, and the overall mechanism of mutual perception and interdependence between cells.
Thousands of years of Chinese medicine practice has proved that "dredging channels and collaterals, making human health".

Considering the orderly accumulation of human cells in meridian system, implantation of artificial materials into human body in interventional medicine will seriously interfere with the orderliness of human cells. There may be problems in this direction of medical development.

Both the ideology of Chinese medicine and western medicine are different but they are complementary. Human body is the unity of material energy and information. Dealing with health not only need the knowledge of structure and function of bio-molecules but also need to know how the energy supplying and information controlling. The current researchers of western medicine pay more attention to the structure and function of gene and proteins but less pay attention to energy and information. A new theory based on the energy supply and information control for holistic medicine is needed in the development of medicine. Scientists should pay attention to both ideology of medicine and develop a new medicine by combining the advantages of both the western medicine and the Chinese medicine [13, $14]$. 


\section{Conclusion}

Respiratory chain is a subject of life science from the perspective of energy. Over the past hundred years, scientific experiments have elucidated the molecular mechanism by which the respiratory chain produces ATP through electronic transmission to provide energy support for living organisms. The discovery of electronic leak bypass reveals the working principle of respiratory chain as a molecular "life-engine". The fact that ROS generated by electronic leak has positive and negative effects reveals that "life-engine" has the ability of self-repairing damage. This property should be the theoretical basis of self-healing medicine. In this paper, the concept of energy entanglement is proposed to describe the evolution of matter and the origin of life in nature. It provides a theoretical basis for explaining the origin of life and lays a foundation for the holistic medical principle based on the function of respiratory chain. Based on the discovery of electronic leak bypass of respiratory chain, this paper explores the new ideas of medical research and emphasizes the position and role of vitality in maintaining health, which is undoubtedly an important conceptual change in medical development.

\section{References}

[1] Yun-Gang Zhao, Zhi-bo Wang and Jian-Xing Xu, Effect of Cytochrome $\mathrm{c}$ on the Generation and Elimination of O2-. and H2O2 in Mitochondria, J. Biol. Chem. 278 (4): 2356-2360 (2003).

[2] Yun-Gang Zhao, and Jian-Xing Xu, The Operation of the Alternative Electron-leak Pathways Mediated by Cytochrome c in Mitochondria. Biochem. Biophys. Rev. Commun. 317: 980-987 (2004).

[3] Zhi-Bo Wang, Yun-Gang Zhao, and Jian-Xing Xu, Cytochrome $\mathrm{c}$ is a Hydrogen Peroxide Scavenger in Mitochondria Protein and Peptide Letters 10: 247-253 (2003).
[4] Jian-xing $\mathrm{Xu}$, Radical Metabolism is Partner to Energy Metabolism in Mitochondria Ann. N. Y. Acad. Sci. 1011: 57-60 (2004).

[5] Lee Min, Xu Jian-xing Detoxifying function of cytochrome c against oxygen toxicity Mitoxhondrion 7 (1-2): 13-16 (2007).

[6] Bielski, B. H., Arudi, R. L. \& Sutherland, M. W. A study of the reactivity of $\mathrm{HO} 2 / \mathrm{O} 2-$ with unsaturated fatty acids. J. Biol. Chem. 258, 4759-4761 (1983).

[7] Chance, B., Sies, H. and Boveris, A. Hydroperoxide metabolism in mammalian organs. Physiol. Rev. 59, 527-605 (1979).

[8] Xin-Jian Wang and Jian-Xing Xu. Salvianic acid A protects human neuroblastoma SH-SY5Y cells against MPP+-induced cytotoxicity, Neuroscience Research, 2005; 51 (2): 129-138.

[9] Xin-Jian Wang and Jian-Xing Xu. Possible involvement of $\mathrm{Ca} 2+$ signaling in rotenone-induced apoptosis in human neuroblastoma SH-SY5Y cells, Neuroscience Letter, 2005; 376 (2): $127-132$.

[10] Xin-Jian Wang, Zhi-Bo Wang and Jian-Xing Xu. Effect of Salvianic acid A on lipid peroxidation and membrane permeability in mitochondria. Journal of Ethnopharmacology, 2005; 97 (3): 441-445.

[11] Suqing Zhanga, Chaoshu Tangb, Jianxing Xua et al"Protective role of 3-nitro-N-methyl-salicylamide on isolated rat heart during 4 hours of cold storage and reperfusion" Transplantation Proceedings 38: 1247-1252 (2006).

[12] Zhi-Bo Wang, Min Li, and Jian-xing Xu, Current Topics in Toxicology, vol. 7, 21-31, (2011).

[13] Xu Jian-xing, The Electron Leak Pathways of Mitochondrial Respiratory Chain and its Potential Application in Medical Research, JSM Cell Dev Biol 3 (1): 1014 (2015).

[14] Jian-xing Xu, Ideas in Scientific Research on Human Health and Heart Care Raised from the Electron Leak Pathways of Mitochondrial Respiratory Chain. J Clin Exp Cardiolog 7: 422. doi: 10.4172/2155-9880.1000422, (2016). 